\title{
Supracricoid Partial Laryngectomy: Oncological and Functional Outcomes
}

\author{
Massimo Mesolella ${ }^{1}$ Brigida lorio ${ }^{1}$ Sarah Buono ${ }^{1}$ Mariano Cimmino ${ }^{1}$ Gaetano Motta ${ }^{2}$ \\ ${ }^{1}$ Department of Neuroscience, Reproductive Sciences, and Dentistry, \\ Università di Napoli Federico II, Napoli, Italy \\ 2 Department of Anesthesiology, Surgical, and Emergency Sciences, \\ Head and Neck Surgery Unit, Università degli Studi della Campania \\ Luigi Vanvitelli, Napoli, Italy

\begin{abstract}
Address for correspondence Massimo Mesolella, MD, PhD, Department of Neuroscience, Reproductive Sciences, and Dentistry, Università di Napoli Federico II, Via Gaetano Filangieri 72, 80121 Napoli, Italy (e-mail: massimo.mesolella@tin.it).
\end{abstract}

Int Arch Otorhinolaryngol 2022;26(1):e75-e84.

\begin{abstract}
\section{Keywords}

- Partial Laryngectomy

- swallowing disorders

- laryngeal cancer

- voice

Introduction In the present study, we have reviewed the outcomes of patients with supracricoid partial laryngectomy (SCPL) in our institution. Our results show that SCPL is a well-tolerated procedure with generally good functional outcomes for patients with advanced laryngeal cancer.

Objective We analyzed the oncological and functional results of a cohort of 35 patients who had undergone SCPL, and we highlighted the complications, identified the overall and disease-free survivals, demonstrating that the reconstructive laryngectomy guarantees the oncological safety and reproducibility of the oncological results, preserving the laryngeal functions and promoting an improvement in the patient's quality of life, favoring communication and interpersonal relationships.

Methods Between 2010 and 2018, 35 patients underwent SCPL for primary and recurrent laryngeal squamous cell carcinomas, and they were divided into two subgroups: in 16 cases, the cricohyoidoepiglottopexy according to the Mayer-Piquet technique was performed, while the remaining 19 cases were submitted to the cricohyoidopexy according to the Labayle technique. In addition to evaluating the oncological results of patients undergoing reconstructive laryngectomy, the present study also aimed to evaluate the functionality of the residual larynx and the quality of life.

Results The overall and disease-free survivals were of $83 \%$ and $76.3 \%$ respectively. All patients were able to swallow. The nasogastric tube was removed after a mean period of 21.8 days (range: 14 to 28 days). The mean decannulation time was of 23.4 days after surgery (range: 15 to 36 days).

Conclusion The curves for the overall and disease-free survivals show that SCPL can guarantee oncological safety comparable to that of total laryngectomies in diseases in the intermediate stage and in carefully-selected advanced stages.
\end{abstract}

received

April 8, 2020

accepted

February 14, 2021

published online

July 19, 2021
DOI https://doi.org/ 10.1055/s-0041-1730020. ISSN 1809-9777. (c) 2021. Fundação Otorrinolaringologia. All rights reserved.

This is an open access article published by Thieme under the terms of the Creative Commons Attribution-NonDerivative-NonCommercial-License, permitting copying and reproduction so long as the original work is given appropriate credit. Contents may not be used for commercial purposes, or adapted, remixed, transformed or built upon. (https://creativecommons.org/ licenses/by-nc-nd/4.0/)

Thieme Revinter Publicações Ltda., Rua do Matoso 170, Rio de Janeiro, RJ, CEP 20270-135, Brazil 


\section{Introduction}

Supracricoid partial laryngectomy (SCPL) with cricohyoidopexy (CHP) was first introduced as an alternative to total laryngectomy (TL) for the treatment of selected cases of glottic and supraglottic cancer by Majer in 1959. In 1974, Piquet described the cricohyoidoepiglottopexy (CHEP) procedure, which is a modification of the Majer technique for glottic carcinomas. ${ }^{1,2}$

The group of partial laryngectomy procedures includes resection of the true and false vocal folds, of both paraglottic spaces, and of the thyroid cartilage. It optionally includes up to one arytenoid cartilage, a portion or all of the epiglottis, and the preepiglottic space. In cases of supraglottic and transglottic cancer, SCPL involves resection of the entire epiglottis and preepiglottic space, and the reconstruction is performed through a CHP. In cases of glottic cancer, SCPL may preserve the epiglottis, and the reconstruction technique is the CHEP.

The supracricoid partial laryngectomy (SCPL) provides a surgical organ-preserving alternative to TL In selected cases of endolaryngeal malignant neoplasms. The advantage of the SCPL over total laryngectomy (TL) is the preservation of lungpowered speech and swallowing function, without permanent stoma, while offering a very high local control rate for selected laryngeal cancers. Local control rates for T2 and T3 laryngeal carcinomas $\geq 90 \%$ have been reported, which are better than those reported for radiation therapy plus chemotherapy and other forms of partial laryngectomy.

As with any technique, the key to a successful outcome following SCPL is careful patient selection. The tumor must be contained within the limits of the en bloc resection area. Clear contraindications include a fixed arytenoid, subglottic extension to the level of the cricoid, hyoid invasion, and extralaryngeal spread. Maintenance of laryngeal function requires the preservation of at least one cricoarytenoid unit (arytenoid cartilage, cricoid cartilage, posterior and lateral cricoarytenoid muscles, and superior and recurrent laryngeal nerves).

\section{Objectives}

a) To analyze the oncological and functional results of a cohort of 35 patients submitted to SCPL, who were consecutively referred to the Department of Neurosciences, Unit of Otolaryngology, Universà di Napoli Federico II, from 2010 to 2018;

b) to highlight complications;

c) to determine the overall and disease-free survivals; and d) to demonstrate that reconstructive laryngectomy guarantees the oncological safety and reproducibility of the oncological results, preserving the laryngeal functions and promoting an improvement in the patient's quality of life, favoring communication and interpersonal relationships.

The Institutional Board of Università di Napoli Federico II approved this retrospective review of medical records and the use of archived data.

\section{Methods}

Between 2010 and 2018, 35 patients underwent SCPL for primary and recurrent laryngeal squamous cell carcinomas. There were 34 males and 1 female, with a median age of 62.6 years (range: 42 to 72 years) (- Table 1 ). The follow-up had a mean duration of 51.4 months (range: 5 to 115 months). All patients underwent the following investigations during the diagnosis:

- fibrolaringoscopy with the aid of video recording;

- radiological investigations (computed tomography [CT] and magnetic resonance imaging [MRI] scans of the neck and thorax) were performed to assess the involvement of the thyroid cartilage, preepiglottic and paraglottic spaces;

- spirometry for the assessment of bronchopulmonary function;

- evaluation of comorbidities for patients at risk; and

- direct microlaryngoscopy and biopsy.

The exclusion criteria were as follows:

- presence of severe chronic obstructive pulmonary disease (FEV $1<50 \%$ );

- presence of severe heart disease ( $\mathrm{FE}<40 \%$ );

- advanced age ( $>75$ years); and

- psychopathy or mental disease.

The absolute contraindications for SLPC were:

- tumors involving the posterior commissure and both arytenoids;

- massive invasion of the lower paraglottic space with infiltration of the submucosa of the piriform sinus; and

- involvement of the retrocricoid area or of the trachea.

The sample was divided into two subgroups according to the surgical modality chosen: group 1 was composed of 16 patients submitted to cricohyoidoepiglottopexy (CHEP) according to the Mayer-Piquet (type-IIa open partial horizontal laryngectomy [OPHL]) technique, while the 19 patients in group 2 underwent cricohyoidopexy (CHP) according the to Labayle (OPHL type IIb) technique. A functional bilateral neck dissection (sparing the accessory nerve, the jugular vein, the sternocleidomastoid muscle, and resecting levels II to IV) was performed in 24 cases, a functional

Table 1 Distribution of the patients according age and gender

\begin{tabular}{|l|l|l|}
\hline Age (years) & $\mathrm{n} / \mathrm{N}$ & Percentage \\
\hline$<55$ & $6 / 35$ & $17.1 \%$ \\
\hline $55-75$ & $28 / 35$ & $80 \%$ \\
\hline$>75$ & $1 / 35$ & $2.9 \%$ \\
\hline Range: $29-76^{*}$ & & \\
\hline Median: $64.6^{*}$ & & \\
\hline Gender & & \\
\hline Male & $34 / 35$ & $97.1 \%$ \\
\hline Female & $1 / 35$ & $2.9 \%$ \\
\hline
\end{tabular}


Table 2 Distribution of patients according the pathologic TNM (pTNM) staging

\begin{tabular}{|l|l|l|l|l|}
\hline pTNM & N0 & N1 & N2 & Total \\
\hline T2 & 8 & 1 & 0 & 9 \\
\hline T3 & 16 & 5 & 0 & 21 \\
\hline T4a & 5 & 0 & 0 & 5 \\
\hline Total & 29 & 6 & 0 & 35 \\
\hline
\end{tabular}

Abbreviation: TNM, tumor, node, metastasis.

ipsilateral neck dissection, in ten cases. Nodal metastases were detected in seven patients, in two cases were bilateral.

None of the patients had been previously submitted to radiation therapy.

The senior surgeon performed all of the SCPL procedures considered in the present study. All patients had squamous cell carcinoma. The postoperative histopathological examination showed resection margins free of tumor in all cases. The distribution of the pT and pN stages is shown in - Table 2.

Neck movement was restricted for two days in the early postoperative period.

All patients monitored for early (local and general) complications and late sequelae were subjected to the same rehabilitation protocol which includes nutritional advice and an assessment through speech therapy.

Rehabilitation began within four days after surgery, with the aim of first educating the patient to take a facilitating position for swallowing (head and neck flexed forward and head rotated to the side). Swallowing exercises were started on the tenth postoperative day. We then proceeded to the tests of swallowing saliva initially, which progressed to swallowing semisolid foods, and then solid nutrition and therefore with liquids. Concomitantly, through the linguobucco-facial praxis, the patient began to emit the first phonemes. When the patient achieved a good level of swallowing regarding solids and liquids, the nasogastric tube was removed. Based on the pathological results (neck metastasis, basaloid features), and in agreement with the oncology and radiotherapy consultants, we considered appropriate to subject eight patients to adjuvant radiotherapy.

The average duration of the follow-up was of 51.4 months (range: 5 to 115 months), and it consisted of an annual fiberoptic laryngoscopy and radiological investigation (CT, MRI, positron emission tomography [PET]). For all patients, the follow-up was monthly in the first year bimonthly in the second year, and quarterly from the third year on.

\section{Functional Evaluation and Performance Status}

During the follow-up, the function of the residual larynx and the quality of life were estimated for 24 patients; the remaining 11 patients were not followed up because, in some cases, the questionnaires had not been completely filled out, and, in other cases, the patients had a low level of schooling, and could not provide the answers, or, had not adhered to the evaluation, not having signed the consent.

For the evaluation of hoarseness, the grade, instability, roughness, breathiness, asthenia, and strain (GIRBAS) scale was used, a semiquantitative scale that enables the assessment, with a score from zero to three, of the extent of the deviance (grade, roughness, breathiness, asthenia, strain, instability) from the norm of vocal characteristics.

In addition, the patients were advised to complete the three following self-assessment questionnaires:

a) M.D. Anderson Dysphagia Inventory (MDADI). ${ }^{3}$ It examines the dysphagia perceived by the patient, and consists of 20 questions and a global assessment (one item). It enables the determination of the emotional (eight items), physical (six items) and functional (five items) factors regarding dysphagia. Each question includes five possible answers (strongly agree; agree; indifferent; disagree; and strongly disagree) which are assigned scores from one to five based on the patient's agreement with the reported statement (the highest and lowest values indicate respectively that the subjectively perceived swallowing as the worst imaginable and completely normal).

b) European Organization for Research and Treatment of Cancer Quality of Life Questionnaire - Head and Neck Module (EORTC QLQ-H\&N35). ${ }^{4}$ The questionnaire is a validated, short instrument based on the cancer-specific measurement of quality of life that analyzes the problems related to the site of the neoplasm (head and neck) and its treatment. It consists of seven parameters:

- pain (items 1, 2, 3, and 4);

- swallowing (items 5, 6, 7, and 8);

- senses problems (items 13 and 14);

- nutrition scale (items 19, 20, 21, and 22);

- speech scale (items 16,23 , and 24);

- social relations (items $18,25,26,27$, and 28); and

- sexuality (items 29 and 30).

There are also ten single questions that evaluate: the presence of dental disease (item 9); the possibility of opening the mouth (item 10); dry mouth (11); dense salivation (item 12); coughing (item 15); uneasiness (item 17); the use of analgesics (item 31); the use of nutritional support (item 32); the use of a feeding tube (item 33); weight lost (item 34); and weight gained (item 35). The answers of the first 30 statements of the QLQ-H\&N35 questionnaire use a four-dimensional scale $(1=$ no; $2=\mathrm{a}$ little; 3 =very; and $4=$ very much); the lower the score, the better the subject's perceptions of their health. For the last five questions, instead, there are two possible answers ( $1=$ no; and 2 =yes), depending on the presence or absence of whatever is being asked.

c) Quality of life after neck dissection. This questionnaire deals with the quality of life in patients undergoing neck dissection; it consists of 12 items, and the first seven evaluate the right and left sides. The factors taken into consideration are:

- stiffness of the shoulder (item 1, right-left);

- neck constriction (item 2, dx-sin); 
- pain (item 3, dx-sin);

- numbness of the neck (item 4, dx-sin);

- falling shoulders (item 5, dx-sin);

- limitation in reaching objects overhead (item 6 , dx$\sin )$;

- neck appearance (item 7, dx-sin);

- swelling of the face (item 8, dx-sin);

- difficulty in dressing (item 9);

- difficulty in combing (item 10);

- reduction in work capacity (item 11); and

- reduction in leisure activities (item 12).

Each of these questions is assigned a score from one to five, in which one indicates the worst situation, and five, the best one.

\section{Statistics and Score Formulas}

\section{Survival}

The average duration of follow-up in the present study was of 51.4 months (range: five to 115 months). The endpoint of the overall survival was the date of death (regardless of the cause), or the date of the last follow-up for patients who were alive. For disease-free survival, patients who died from causes not related to laryngeal cancer were excluded on the date of their death. The endpoint of the disease-free survival was the date of recurrence. The MedCalc (MedCalc Software Ltd., Ostend, Belgium) software, version 12.5.0.0, was used to calculate the overall and disease-free survival using Kaplan-Meir curves. ${ }^{5}$

\section{MDADI}

The most frequently used method in the literature for the evaluation of the MDADI dysphagia questionnaire is to transform the data obtained in a score from 0 to 100 . The formula is to multiply by 20 the score obtained on the global assessment question; as for the emotional, physical and functional factors, which consist of several items, the average is calculated and multiplied by $20 .^{6}$

\section{EORTC QLQ-H\&N35}

The method used to read the data obtained from the EORTC QLQ-H\&N35 is the raw score, which is described in the Manual Scoring (3rd edition, 2001) proposed by the EORTC. ${ }^{7}$ A linear transformation was then applied to this value to obtain the final score, in percentages that range from zero to 100.

\section{Quality of Life after Neck Dissection}

The raw data obtained were transformed into a score from zero to 100 , as indicated in the literature, multiplying the score of the question by 20. For items 1 to 7 , the average of the values obtained regarding the patients subjected to functional bilateral neck dissection was calculated; in case of functional unilateral neck dissection, only the affected side was considered. The only patient who did not undergo emptying of the lymph node of the neck was not included in the study, as he underwent a TL The same evaluation was also used for the arm test.

To assess the reliability of the questionnaires and of each extrapolated dimension, an analysis was performed using Cronbach's $\alpha,{ }^{5}$ a coefficient that measures the internal consistency of item groupings. High values of $\alpha$ indicate that the subjects examined express a coherent attitude regarding each item belonging to the grouping. Values above 0.70 are considered acceptable in the literature. ${ }^{5}$ The results concerning Cronbach's $\alpha$ were obtained with the help of the MedCalc program (version 12.5.0.0).

\section{Results}

\section{Acute Complications and Late Sequelae}

None of the patients died in the immediate postoperative period. Laryngeal necrosis and ruptured pexy were not observed.

In $3 / 35(8.5 \%)$ patients, it was necessary to perform an endoscopic reduction of edema or arytenoid mucosal with $\mathrm{CO} 2$ laser for the appearance of postoperative dyspnea dysphagia or respiratory. The procedure was performed on average 6 months (range: three to 12 months) after the SCPL. In one case, this procedure was bilateral, and the tracheotomy was performed again (the patient had already been decannulated). The most frequently reported complication from these procedures was aspiration pneumonia, which occurred in $6 / 35$ (17\%) of our patients. Pneumonia often occurred (in 3/35 (14,2\%) patients in our sample) not during hospitalization, but within six months of the discharge from the hospital, and it was managed successfully with antibiotic therapy.

\section{Oncological Results}

The follow-up for the surviving patients was of 61.4 months on average. The overall and disease-free survivals were of $83 \%$ and $76.3 \%$ respectively. - Figs. 1 and $\mathbf{2}$ show the survival curves obtained using the Kaplan-Meier method depending on the type of surgery compared with TLs performed in our department in the same period.

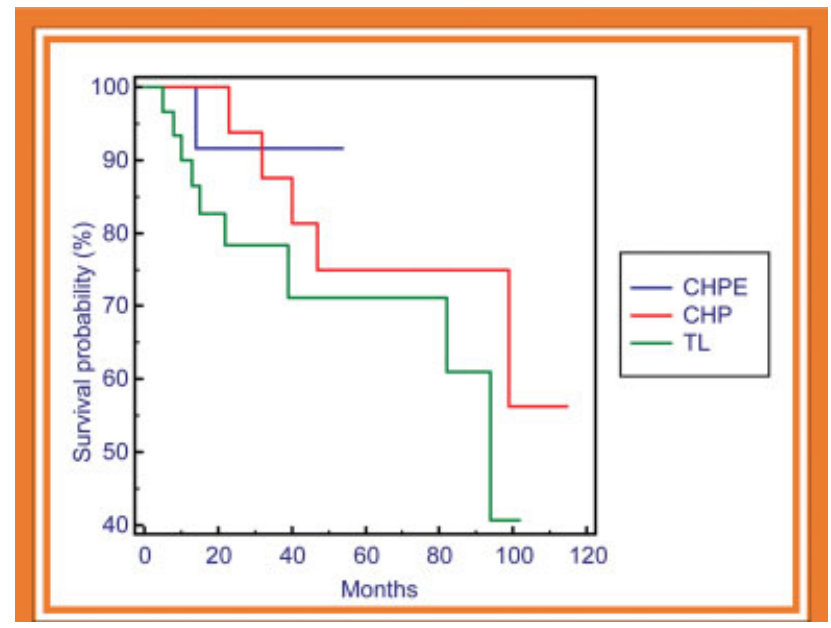

Fig. 1 Kaplan-Meier curve: overall survival. 


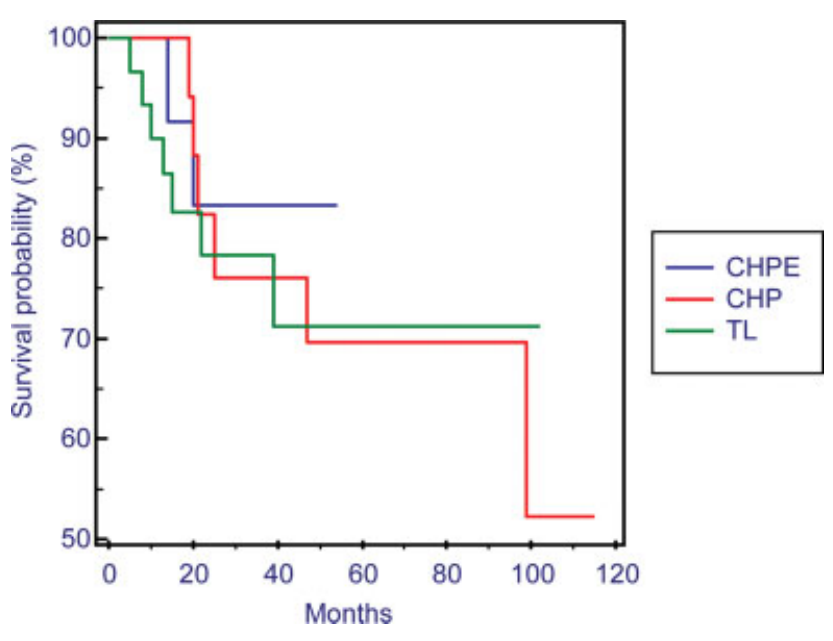

Fig. 2 Kaplan-Meier curve: disease-free survival.

Considering the overall survival based on the surgical technique, death occurred in only one patient operated with Majer-Piquet technique (CHEP), and in five patients submitted to the Labayle technique (CHP).

The disease-free survival curves based on the tumor stage in SCPL patients (-Fig. 2 ) show the presence of recurrence in one $(2,8 \%)$ stage-II patient 99 months ( 8 years) postoperatively. Within 47 months (almost four years), there were five $(14,2 \%)$ cases of relapse to stage III; and three $(8,5 \%)$ cases of relapse to stage IVA occurred almost 18 months postoperatively. Therefore, patients in advanced stages are burdened with a greater and earlier risk of recurrence.

The tumor recurred in nine $(25,7 \%)$ patients who underwent SCPL (three with the CHEP and six with CHP technique). In six $(17.1 \%)$ of these cases, TL was performed, the other three $(8.6 \%)$ died following the reappearance of the tumor.

\section{Functional Results}

All patients were able to swallow. The nasogastric tube was removed after an average of 21.8 days (range: 14 to 28 days).

The tracheal cannula was removed, allowing the tracheotomy to close, rarely with tracheal plastic surgery. The mean decannulation time was of 23.4 days after surgery (range: 15 to 36 days). The tracheal cannula is still in place in one case, $1 / 35$ ( $2 \%$ ), because the patient chose not to proceed with the removal.

In our cases series, we observed that sparing both arytenoids did not improve the functional results, but we found that the patient in whom they were spared had a much shorter period of rehabilitation than the patients in whom only one arytenoid was preserved. Regarding the duration of the rehabilitation, no differences were recorded between the patients who underwent CHEP and those treated with CHP.

\section{Quality of Life}

The function of the residual larynx and the quality of life of 24 (68.5\%) out of the 35 patients were estimated because, in some cases, the questionnaires had not been completely filled out; in other cases, the patients had a low level of schooling, and were not able to provide the answers; more-
Table 3 GIRBAS Scale: Median and Standard Deviation

\begin{tabular}{|l|l|l|l|l|l|l|}
\hline & G & I & R & B & A & S \\
\hline Median & 2,13 & 2,08 & 2,13 & 2,25 & 2,17 & 2,17 \\
\hline DS & 0,61 & 0,78 & 0,74 & 0,68 & 0,64 & 0,76 \\
\hline
\end{tabular}

Abbreviation: G, grade; I, instability; R, roughness; B, breathiness; A, asthenia; S, strain.

over, there were patients who did not adhere to the evaluation, and did not sign the consent.

\section{GIRBAS Scale}

None of the patients scored zero in any of the parameters in the GIRBAS scale. In total, 15 out of the 24 (62\%) patients examined scored 2 on the grade $(G)$ of dysphonia. Regarding instability $(\mathrm{I})$, roughness (R), breathiness (B), and strain $(\mathrm{S})$, most patients, $20 / 24$ (83\%), obtained scores varying from two to three, which are considered medium to high. As for asthenia (A), most patients $14 / 24$ (58\%) scored two. Overall, the data show a severely dysphonic voice for all patients who underwent SCPL.

- Table 3 and -Fig. 3 show the average and standard deviation (SD) values for each parameter analyzed by the scale.

\section{Score on the MDADI}

Considering the score on the global assessment, there were patients from the sample classified in every range, with a slight prevalence of the range from $50 \%$ to $75 \%$, which indicates a swallowing capacity perceived as medium to good. The emotional (embarrassment in eating, going out with friends etc.) and functional factors (problems in swallowing limit personal and social life) had little influence on the quality of life of patients, since most of them scored $\sim$ $75 \%$ on the questionnaires regarding these factors. On the other hand, the physical factors (effort in swallowing, coughing with liquids) had more influence, for most of them scored in the range from $50 \%$ to $75 \%$. Based on the analysis of the data, it is worth mentioning the statement "coughing when trying to drink liquids" (P8) among the items included in the physical factors, in which almost all patients had medium or low scores. Taking all the parameters into account, swallowing, although compromised, seems to be perceived as medium to good by the patients, with most of them scoring in the range from $60 \%$ to $72.5 \%$. (- Table 4 , - Table 5)

The Cronbach $\alpha$ coefficient calculated for the emotional, functional and physical factors was of $0.90,0.82$, and 0.95 respectively; analyzing the MDADI items, overall, the value obtained was 0.95 . The internal consistency, and, therefore, the reliability of the questionnaire, was considered high.

\section{EORTC QLQ-H\&N35}

Unlike the MDADI, a low score on the EORTC QLQ-H\&N35 corresponds to a better health condition perceived by the patient (-Table $\mathbf{6}$ ).

In general, the patients fell into the medium or low ranges (lower than 50\%). The exceptions are the results relating to 


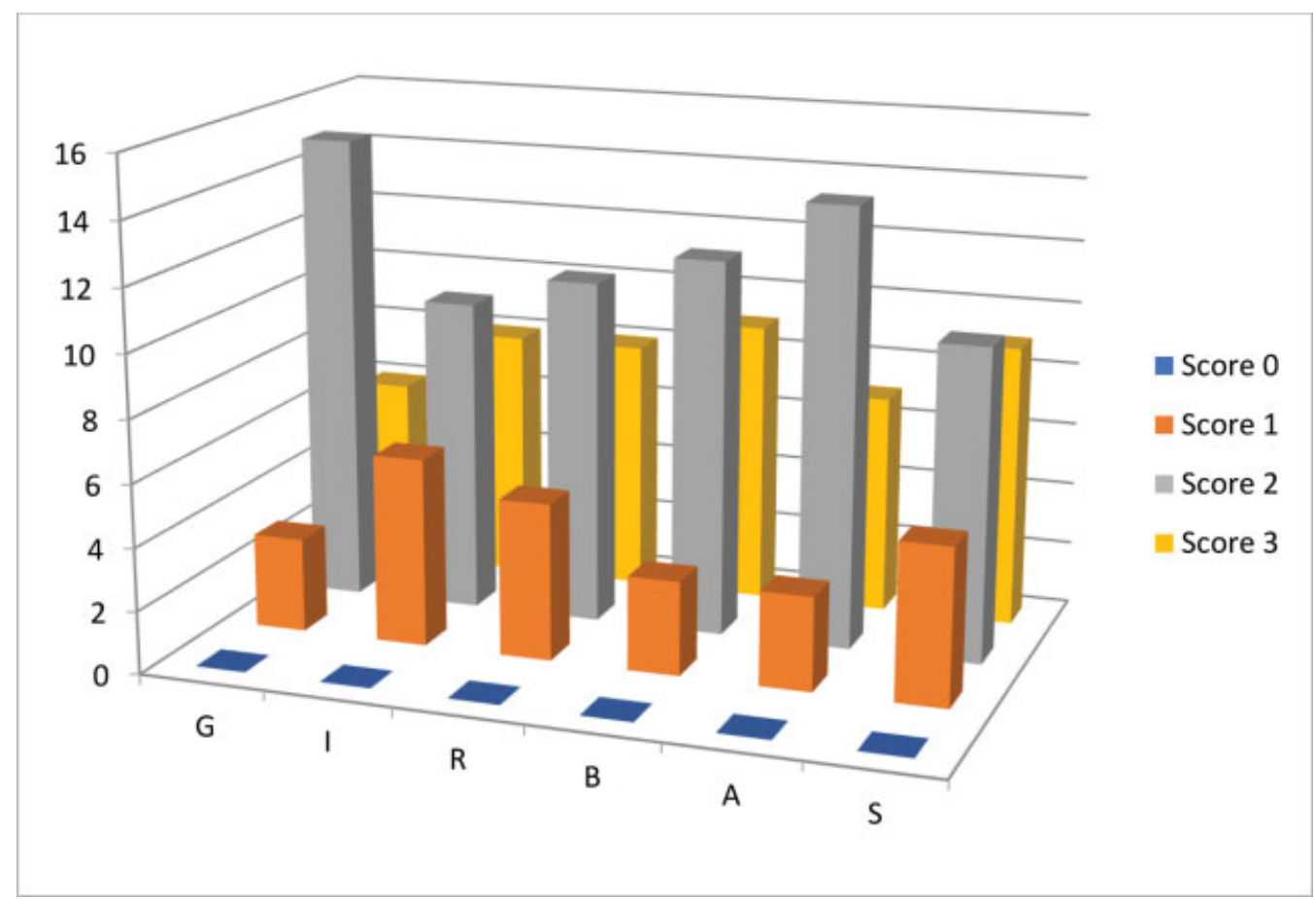

Fig. 3 Scores of the patients on the GIRBAS scale.

swallowing, social relationships and sexuality, which present a greater variability of distribution among the different score ranges. Based on the analysis of the results, coughing (after ingesting liquids) and communication problems are the factors that patients consider most likely to affect their quality of life. The raw data show that, on items 23 (difficulty in talking to other people) and 24 (difficulty in talking on the phone), the patients almost always had medium or high scores. Among the other factors that worsen the quality of life, there are the problems in swallowing and the difficulty in social relationships. - Table 7 shows the average and standard deviation of the various parameters.

The Cronbach $\alpha$ coefficient was calculated for the parameters that included several items: pain -0.80 ; swallowing -
0.86; senses problems (smell and taste) - 0.79; communication -0.90 ; eating in public -0.78 ; social relations -0.91 ; sexuality - 0.95; overall score on the EORTC QLQ-H\&N350.96 . All values were higher than 0.70 , and the questionnaire waconsidered reliable.

\section{Quality of Life after Neck Dissection}

Shoulder function is one of the most studied quality of life outcomes, given its potential to result in significant morbidity and interference in many activities of daily living. The questions focus on neck or shoulder pain, stiffness, difficulty with self-care, ability to lift objects or reach overhead, overall activity level, participation in social activities, ability to participate in leisure or recreational activities, and ability

Table 4 M.D. Anderson Dysphagia Inventory Score

\begin{tabular}{|c|c|c|c|c|c|}
\hline Score & Minimum & $25 \%$ & $50 \%$ & $75 \%$ & Maximum \\
\hline Global assessment & 5 & 3 & 8 & 5 & 3 \\
\hline Emotional factors & 1 & 3 & 5 & 10 & 5 \\
\hline Functional factors & 0 & 4 & 7 & 9 & 4 \\
\hline Physical factors & 0 & 2 & 8 & 8 & 6 \\
\hline
\end{tabular}

Table 5 Median and standard deviation values on the M.D. Anderson Dysphagia Inventory Score

\begin{tabular}{|l|l|l|l|l|}
\hline & Global assessment & Emotional factors & Functional factors & Physical factors \\
\hline Median & 60 & 72.5 & 70.84 & 65.5 \\
\hline Standard deviation & 27.66 & 21.92 & 19.6 & 24.4 \\
\hline
\end{tabular}


Table 6 Distribution of patients according to the EORTC QLQ-H\&N35

\begin{tabular}{|l|l|l|l|l|l|}
\hline Score & Minimum & $\mathbf{2 5} \%$ & $\mathbf{5 0}$ & $\mathbf{7 5 \%}$ & Maximum \\
\hline Pain & 18 & 3 & 2 & 1 & 0 \\
\hline Swallowing & 5 & 9 & 5 & 5 & 0 \\
\hline Dental disease & 14 & 6 & 1 & 2 & 1 \\
\hline Opening of the mouth & 14 & 7 & 1 & 2 & 0 \\
\hline Dry mouth & 7 & 7 & 1 & 8 & 1 \\
\hline Dense salivation & 11 & 10 & 1 & 2 & 0 \\
\hline Sense & 12 & 4 & 7 & 1 & 0 \\
\hline Coughing & 1 & 7 & 0 & 13 & 3 \\
\hline Uneasiness & 14 & 5 & 2 & 3 & 0 \\
\hline Social role of food & 11 & 6 & 6 & 1 & 0 \\
\hline Communication & 3 & 2 & 8 & 8 & 3 \\
\hline Social relations & 7 & 8 & 6 & 2 & 1 \\
\hline Sexuality & 5 & 6 & 4 & 3 & 6 \\
\hline Analgesics & 19 & - & - & - & 5 \\
\hline Nutritional support & 24 & - & - & - & 0 \\
\hline Nutritional tube & 24 & - & - & - & 0 \\
\hline Weight gain & 14 & - & - & - & 10 \\
\hline Weight loss & 10 & - & - & - & 14 \\
\hline
\end{tabular}

Abbreviation: EORTC QLQ-H\&N35, European Organization for Research and Treatment of Cancer Quality of Life Questionnaire - Head and Neck Module.

Table 7 Median and standard deviation values on the EORTC QLQ-H\&N35

\begin{tabular}{|l|l|l|}
\hline & Median & Standard deviation \\
\hline Pain & 18.5 & 17.23 \\
\hline Swallowing & 39.3 & 25.03 \\
\hline Dental disease & 23.2 & 30.1 \\
\hline Opening the mouth & 14.4 & 20.3 \\
\hline Dry mounth & 37.2 & 29.5 \\
\hline Dense salivation & 19.5 & 22.7 \\
\hline Sense problems & 23.4 & 22.9 \\
\hline Coughing & 57.6 & 22.8 \\
\hline Uneasiness & 16.2 & 23.8 \\
\hline Social role of food & 26.3 & 21.7 \\
\hline Comunication & 54.8 & 22.8 \\
\hline Social Relations & 30.1 & 22.8 \\
\hline Sexuality & 52.1 & 33.8 \\
\hline Analgesics & 21 & 42.2 \\
\hline Nutritional support & 0 & 0.00 \\
\hline Nutritional tube & 0 & 0.00 \\
\hline Weight gain & 26 & 45.3 \\
\hline Weight loss & 56.1 & 52.7 \\
\hline
\end{tabular}

Abbreviation: EORTC QLQ-H\&N35, European Organization for Research and Treatment of Cancer Quality of Life Questionnaire - Head and Neck Module.

to work. The data obtained show that there has been a minimal reduction in the activities of daily living, as well as in activities related to work. The limitation in reaching high objects was the factor with the worst score (-Table 8, - Table 9).

\section{Discussion}

The most recent approach in the treatment of laryngeal tumors aims not only at guaranteeing safety from oncologic perspective, but also at obtaining an improvement in the quality of life of the patient by favoring breathing and swallowing through natural routes and an acceptable quality of voice for the preservation of social relations. ${ }^{6-10}$ Since the early 1990s, organ preservation protocols have been developed, which involve the administration of chemotherapeutic drugs in conjunction with radiation therapy. ${ }^{11,12}$ At the same time, in the surgical field, we have tried to develop techniques to preserve part of the laryngeal structure, guaranteeing the preservation of the sphincter and respiratory function of the larynx with equal oncological results to those of TL. ${ }^{13-15}$ Currently, as regards the treatment of glottic and supraglottic tumors in the intermediate to advanced stages, SCPL is the surgical procedure that has received unanimous approval regarding the oncological and functional results. ${ }^{15-18}$ In our case series, the overall and disease-free survivals were of $83 \%$ and $76.3 \%$ respectively. Recurrences occurred in nine $(25.7 \%)$ out of 35 patients. 
82 Supracricoid Partial Laryngectomy Mesolella et al.

Table 8 Quality of life after neck dissection

\begin{tabular}{|l|l|l|l|l|l|}
\hline Stiffness of the shoulder & 6 & 6 & 5 & 4 & 3 \\
\hline Neck constriction & 3 & 4 & 8 & 8 & 1 \\
\hline Pain & 7 & 7 & 6 & 3 & 1 \\
\hline Numbness of the neck & 6 & 7 & 5 & 4 & 2 \\
\hline Falling shoulders & 4 & 4 & 5 & 4 & 7 \\
\hline Limitation in reaching objects overhead & 8 & 5 & 5 & 4 & 2 \\
\hline Neck appearance & 3 & 3 & 4 & 8 & 6 \\
\hline Edema of the face & 3 & 4 & 5 & 7 & 5 \\
\hline Difficulty in dressing & 3 & 2 & 7 & 8 & 4 \\
\hline Difficulty in combing & 7 & 6 & 6 & 3 & 2 \\
\hline Reduction in work ability & 2 & 3 & 6 & 7 & 6 \\
\hline Reduction in leisure activities & 2 & 2 & 6 & 6 & 8 \\
\hline
\end{tabular}

Swallowing function is the main problem in the early postoperative period. ${ }^{19-22}$ Thus, it requires time and the involvement of a speech pathologist for this function to be restored. All patients were able to swallow. The nasogastric tube was removed after a mean period of 21.8 days (range: 14 to 28 days).

The tracheal cannula was removed, enabling the closure of the tracheotomy, which was rarely performed through tracheal plastic surgery. The mean decannulation time was of 23.4 days after surgery (range: 15 to 36 days). Only in one (3.5\%) case the tracheal cannula is still in place, because the patient chose not to proceed with the removal. ${ }^{23}$ We tend to prefer decannulation after deglutition without aspiration to minimize the risk of serious pulmonary complications posed by early postoperative aspiration.

In three $(8.5 \%)$ patients, it was necessary to perform an arytenoid mucosal reduction of edema in suspension microlaryngoscopy (SML) with $\mathrm{CO}_{2}$ laser.

Table 9 Quality of life after neck dissection: median and standard deviation

\begin{tabular}{|l|l|l|}
\hline & Median & $\begin{array}{l}\text { Standard } \\
\text { deviation }\end{array}$ \\
\hline Stiffness of the shoulder & 53.4 & 27.4 \\
\hline Neck constriction & 60 & 22.06 \\
\hline Pain & 46.6 & 23.34 \\
\hline Numbness of the neck & 50.8 & 25.6 \\
\hline Falling shoulders & 65 & 29.6 \\
\hline $\begin{array}{l}\text { Limitation in } \\
\text { reaching objects overhead }\end{array}$ & 49.2 & 27 \\
\hline Neck appearance & 69.2 & 27 \\
\hline Edema of the face & 65.8 & 26.6 \\
\hline Difficulty in dressing & 66.6 & 24.8 \\
\hline Difficult in combing & 49.2 & 25.6 \\
\hline Reduction in work ability & 70 & 25 \\
\hline Reduction in leisure activities & 74 & 26 \\
\hline
\end{tabular}

In addition to evaluating the oncological results of patients undergoing SCPL, the present study also aimed at evaluating the functionality of the residual larynx and the quality of life through the GIRBAS Scale regarding the perceptual evaluation of the vocal emission, and through two self-assessment questionnaires : the MDADI, to assess patient-perceived dysphagia, and the EORTC QLQ-H\&N35, to obtain cancer-specific measurements of quality of life by analyzing problems related to the site of the neoplasm (head and neck) and its treatment. The scores of the patients were medium to high scores on the instability (I), roughness (R), breathiness (B), and strain (S) domains; but there was a prevalence of average scores on the grade $(G)$ of dysphonia and asthenia (A) domains. The voice was severely dysphonic in every patient in the sample.

The function of the residual larynx and the quality of life of 24 patients were assessed This assessment did not include the 35 patients in the sample because, in some cases, the questionnaires had not been completely filled out; in other cases, the patients had a low level of schooling, and were not able to provide the answers; and some patients did not adhere to the evaluation, and did not sign the consent.

As the questionnaires had no translation to Italian, they had to be translated before they were administered to the patients. In the case of the MDADI, in addition to the translation, two items (E7 and F2) were modified, which, in the official version, provided a reverse score compared with the others. We decided to do so to simplify the operations of counting the final score, considering the use of the Cronbach $\alpha$ coefficient to evaluate the internal consistency of the parameters with more items and the reliability of the questionnaire. Based on the analysis of the data, the global assessment of dysphagia was perceived by the patients as medium to good (average of 60\%).

Emotional and functional factors have little influence on the quality of life of the patients, since most of them scored in the $75 \%$ range on these sections of the questionnaires. Physical factors seem to have more influence (average of $65 \%)$. Regarding the analysis of the raw data, we must mention the statement "Coughs when trying to drink 
liquids" (P8) among the items of Physical factors, in which almost every patient had average or low scores. Taking all the parameters into account, swallowing, although compromised, seems to be perceived as medium to good by the patients (regarding the function, the postoperative scores ranged from $60 \%$ 65\% compared with the preoperative scores). The Cronbach $\alpha$ coefficient was always above 0.70 , which enables us to consider the questionnaire reliable.

The quality of life was assessed through the EORTC QLQ$H \& N 35$, and the results were expressed based on a scale from 0 to 100 . Unlike the MDADI, a low score corresponds to a better health condition perceived by the patient. In general, the scores of the different items fell in the medium to low range (lower than 50\%). The factors considered by the patients to be most influential on the quality of life are:

- coughing (after swallowing liquids);

- the problems inherent to communication, which impact the maintenance of normal social relations;

- reports concerning swallowing.

The Cronbach $\alpha$ coefficient was calculated for the parameters that included several items: pain - 0.80; swallowing 0.86 ; sense problems -0.79 ; communication -0.90 ; eating in public - 0.78 ; social relations - 0.91 ; sexuality -0.95 , overall score on the EORTC QLQ-H\&N35-0.96. All values were higher than 0.70 ; therefore, the questionnaire is considered reliable.

\section{Conclusion}

The overall and disease-free survival curves show that SCPL can guarantee oncologic safety comparable to that of TLs performed in patients with diseases in intermediate stages (II, III), and in carefully selected advanced stage cases (IV A).

With the SCPL surgeons are able to avoid a demolitive and non-functional intervention, enabling a subsequent restoration of the voice and swallowing without a definitive tracheostoma. Swallowing is the main problem in the early postoperative period, amd it takes a certain time to recover in most cases. The voice after SCPL remains a problem, because it is deep, rough and one cannot tell the speaker's gender just by hearing it. Therefore, intensive rehabilitation and a close follow-up program are the key points for a successful outcome, and to maintain a satisfactory quality of life. The results obtained from our study lead us to affirm that, in selected patients, SCPL is a valid alternative to TL, for it enables excellent safety from an oncologic perspective and the avoidance of the functional disadvantages and the issues regarding social relations that are linked to definitive tracheostoma.

\section{Conflict of Interests}

The authors have no conflict of interests to declare.

\section{References}

1 Piquet JJ, Darras JA, Berrier A, Roux X, Garcette L. Les laryngectomies subtotales fonctionnelles avec CHP. Ann Otolaryngol 1986; 103:411-415
2 Labayle J, Bismuth R. Total laryngectomy with reconstitution. Ann Otolaryngol Chir Cervicofac 1971;88(04):219-228

3 Chen AY, Frankowski R, Bishop-Leone J, et al. The development and validation of a dysphagia-specific quality-of-life questionnaire for patients with head and neck cancer: the M. D. Anderson dysphagia inventory. Arch Otolaryngol Head Neck Surg 2001;127 (07):870-876

4 Singer S, Wollbrück D, Wulke C, et al. Validation of the EORTC QLQ-C30 and EORTC QLQ-H\&N35 in patients with laryngeal cancer after surgery. Head Neck 2009;31(01):64-76

5 Heo M, Kim N, Faith MS. Statistical power as a function of Cronbach alpha of instrument questionnaire items. BMC Med Res Methodol 2015;15:86

6 Chevalier D, Piquet JJ. Subtotal laryngectomy with cricohyoidopexy for supraglottic carcinoma: review of 61 cases. Am J Surg 1994;168(05):472-473

7 de Vincentiis M, Minni A, Gallo A. Supracricoid laryngectomy with cricohyoidopexy (CHP) in the treatment of laryngeal cancer: a functional and oncologic experience. Laryngoscope 1996;106(9 Pt 1):1108-1114

8 Laccourreye O, Brasnu D, Périé S, Muscatello L, Ménard M, Weinstein G. Supracricoid partial laryngectomies in the elderly: mortality, complications, and functional outcome. Laryngoscope 1998;108(02):237-242

9 Naudo P, Laccourreye O, Weinstein G, Hans S, Laccourreye H, Brasnu D. Functional outcome and prognosis factors after supracricoid partial laryngectomy with cricohyoidopexy. Ann Otol Rhinol Laryngol 1997;106(04):291-296

10 Brasnu D, Menard M, Fabre A, Janot F, Laccourreye H. [Partial supracricoid laryngectomies: techniques, indications and results]. J Otolaryngol 1988;17(04):173-178

11 Laccourreye H, Laccourreye O, Weinstein G, Menard M, Brasnu D. Supracricoid laryngectomy with cricohyoidopexy: a partial laryngeal procedure for selected supraglottic and transglottic carcinomas. Laryngoscope 1990;100(07):735-741

12 Laccourreye O, Laccourreye L, Muscatello L, Périé S, Weinstein G, Brasnu D. Local failure after supracricoid partial laryngectomy: symptoms, management, and outcome. Laryngoscope 1998;108 (03):339-344

13 Crevier-Buchman L, Laccourreye O, Weinstein G, Garcia D, Jouffre V, Brasnu D. Evolution of speech and voice following supracricoid partial laryngectomy. J Laryngol Otol 1995;109(05):410-413

14 Laccourreye O, Crevier-Buchmann L, Weinstein G, Biacabe B, Laccourreye H, Brasnu D. Duration and frequency characteristics of speech and voice following supracricoid partial laryngectomy. Ann Otol Rhinol Laryngol 1995;104(07):516-521

15 Gong $\mathrm{H}$, Zhou L, Wu H, et al. Long-term clinical outcomes of supracricoid partial laryngectomy with cricohyoidoepiglottopexy for glottic carcinoma. Acta Otolaryngol 2019;139(09): 803-809

16 Crosetti E, Bertolin A, Molteni G, et al. Patterns of recurrence after open partial horizontal laryngectomy types II and III: univariate and logistic regression analysis of risk factors. Acta Otorhinolaryngol Ital 2019;39(04):235-243

17 Succo G, Fantini M, Rizzotto G. Supratracheal partial laryngectomy: indications, oncologic and functional results. Curr Opin Otolaryngol Head Neck Surg 2017;25(02):127-132

18 Succo G, Crosetti E, Bertolin A, et al. Benefits and drawbacks of open partial horizontal laryngectomies, Part A: Early- to intermediate-stage glottic carcinoma. Head Neck 2016;38(Suppl 1): E333-E340

19 Succo G, Crosetti E, Bertolin A, et al. Benefits and drawbacks of open partial horizontal laryngectomies, Part B: Intermediate and selected advanced stage laryngeal carcinoma. Head Neck 2016;38 (Suppl 1):E649-E657

20 Rizzotto G, Crosetti E, Lucioni M, Succo G. Subtotal laryngectomy: outcomes of 469 patients and proposal of a comprehensive and 
84 Supracricoid Partial Laryngectomy Mesolella et al.

simplified classification of surgical procedures. Eur Arch Otorhinolaryngol 2012;269(06):1635-1646

21 Succo G, Peretti G, Piazza C, et al. Open partial horizontal laryngectomies: a proposal for classification by the working committee on nomenclature of the European Laryngological Society. Eur Arch Otorhinolaryngol 2014;271(09):2489-2496
22 Motta S, Cesari U, Mesolella M, Motta G. Functional vocal results after $\mathrm{CO} 2$ laser endoscopic surgery for glottic tumours. J Laryngol Otol 2008;122(09):948-951

23 Mesolella M, Iorio B, Misso G, et al. Role of perineural invasion as a prognostic factor in laryngeal cancer. Oncol Lett 2016;11(04): 2595-2598 